\title{
Design, performance, and analysis of a measurement of optical properties of antarctic ice below $400 \mathrm{~nm}$
}

\section{The IceCube Collaboration}

(a complete list of authors can be found at the end of the proceedings)

E-mail: jannes.brostean-kaiser@icecube.wisc.edu

The IceCube Neutrino Observatory, located at the geographic South Pole, is the world's largest neutrino telescope, instrumenting $1 \mathrm{~km}^{3}$ of Antarctic ice with 5160 photosensors to detect Cherenkov light. For the IceCube Upgrade, to be deployed during the 2022-23 polar field season, and the enlarged detector IceCube-Gen2 several new optical sensor designs are under development. One of these optical sensors, the Wavelength-shifting Optical Module (WOM), uses wavelength-shifting and light-guiding techniques to measure Cherenkov photons in the UV range from $250 \mathrm{~nm}$ to $380 \mathrm{~nm}$. In order to understand the potential gains from this new technology, a measurement of the scattering and absorption lengths of UV light was performed in the SPICEcore borehole at the South Pole during the winter seasons of 2018/2019 and 2019/2020. For this purpose, a calibration device with a UV light source and a detector using the wavelength shifting technology was developed. We present the design of the developed calibration device, its performance during the measurement campaigns, and the comparison of data to a Monte Carlo simulation.

Corresponding authors: Jannes Brostean-Kaiser ${ }^{1 *}$

${ }^{1}$ DESY Zeuthen, D-15738 Zeuthen

* Presenter

$37^{\text {th }}$ International Cosmic Ray Conference (ICRC 2021)

July 12th - 23rd, 2021

Online - Berlin, Germany 


\section{Introduction / Wavelength-shifting Optical Module}

The IceCube Neutrino Observatory is a cubic-kilometer detector installed in the ice at the geographic South Pole at depths between $1,450 \mathrm{~m}$ and 2,450 $\mathrm{m}$ [1]. The detector was completed in 2010. To reconstruct direction, energy, and flavor of interacting neutrinos the Cherenkov radiation, emitted by charged secondary particles, is measured.

To improve the reconstruction of low energy neutrinos and the calibration of the instrumented ice, the IceCube Upgrade will be deployed in the austral summer 2022-2023. Seven additional strings will be deployed, including several types of novel optical modules.

Several of the new modules under development, are designed to measure Cherekenkov radiation in the UV range. This improves the sensitivity of the modules since the number of emitted Cherenkov photons is proportional to one over the wavelength squared.

One of these UV-sensitive detectors is the Wavelength-shifting Optical Module (WOM)[2]. The WOM consists of a $76 \mathrm{~cm}$ long transparent (PMMA or quartz glass) tube with $10.6 \mathrm{~cm}$ diameter. The tube is coated with a wavelength-shifting paint [3] and connected to two photomultiplier tubes (PMTs), one on each side. The paint absorbs photons with a wavelength between $250 \mathrm{~nm}$ and $400 \mathrm{~nm}$ and reemits them at roughly $420 \mathrm{~nm}$. The reemitted photons are guided via total internal reflection to one end of the tube and are detected by the PMTs.

\section{Ice Properties}

To understand the potential improvement of new optical modules, the surrounding material has to be calibrated in the sensitive range. The Antarctic ice originates in compacted snow turning to ice over long times. To measure scattering and absorption specifically, an in-situ measurement device, the UV calibration device (UV logger) has been built.

\subsection{Absorption}

In the visible spectrum down to $300 \mathrm{~nm}$, the ice is mostly transparent, with absorption and scattering driven by impurities in the ice like dust, mineral, or soot [4]. In the very deep UV range a strong absorption occurs, the "Urbach tail" [5]. The exact cutoff wavelength is yet unknown but believed to be below $200 \mathrm{~nm} \mathrm{[6].}$

\subsection{Scattering}

Using the AMANDA detector the scattering and absorption coefficient could be calibrated down to $337 \mathrm{~nm}$. Above $1300 \mathrm{~m}$ depth the scattering is dominated by small air bubbles converting to craigite in the IceCube depth range due to the ice pressure [7]. Below this so-called bubbledominated region, the photons scatter on aforementioned impurities. The particles have varying radii between a few nanometers and several micrometer [4], which results in a mixture of Rayleigh and Mie-Scattering.

\section{In-situ measurement in the SPICEcore hole}

The in-situ measurements were done in the South Pole ice core hole (SPICEcore hole). It is an open borehole at about $1 \mathrm{~km}$ distance from the IceCube array with a depth of $1750 \mathrm{~m}$ [8] and 126 


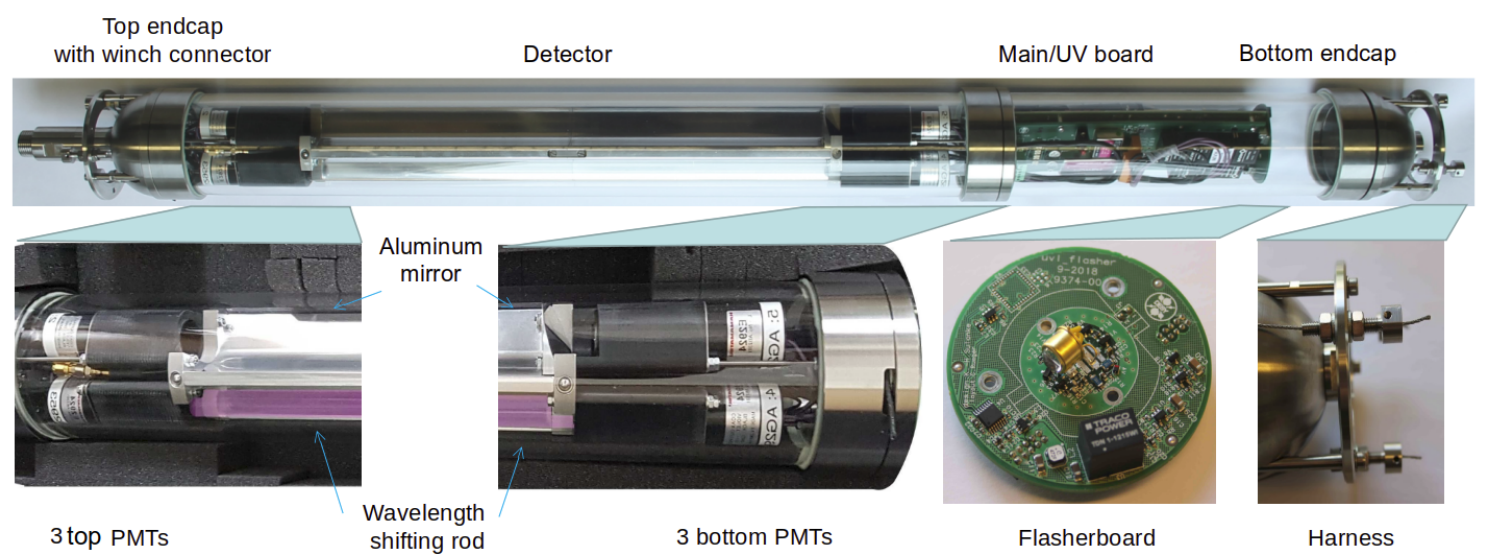

Figure 1: UV Calibration device with a detector, using PMTs, two open ones and four connected to wavelength shifting rods, a light source, capable of pulsing light with nanosecond pulse width and the read out electronic, stored in a quarz glass vessel with titanium endcaps and flanges .

mm diameter. During the drilling process, the hole was filled with Estisol 140, a synthetic ester fluid that stays liquid in the South Pole environment. As its density is very similar to the surrounding ice, it prevents the hole from collapsing and keeps the hole open for calibration measurements.

To measure in an open hole, the measurement device has to be the light emitter and detector at the same time. The light is sent out into the ice in nanosecond short pulses. The detector records the arrival time of the back-scattered photons. This time distribution can later be compared to simulation to obtain the ice properties. Early simulations suggest that the rising edge of the distribution is driven by the scattering coefficient, while the tail of the distribution is driven by the absorption coefficient. These effects are visible in the Figures 5 a) and b).

Since a measurement with emitter and detector at the same place is more sensitive to backward scattering than forward scattering, an additional future task will be the comparison between this scattering measurement and former measurements with large detectors as IceCube or AMANDA.

In addition to the UV Calibration device several other in-situ measurements took place in the two seasons as the Luminescence Logger [9], the Camera System [10] and the dust logger [11].

\section{Optimized UV calibration device}

The device, designed for this measurement consists of a LED-based light source with different wavelengths and a UV-sensitive detector. The detector is divided longitudinally into three segments by aluminum mirrors. Two PMTs are placed in every segment (six in total), one near the light source (bottom) and one on the top. In the two segments opposite of the LED, PMMA rods of $50 \mathrm{~cm}$ length and $2 \mathrm{~cm}$ diameter are connected to the PMTs. The rods are coated with a wavelength shifting paint, developed for the WOM. In the segment facing the same direction as the LED the PMTs are left open for direct photon detection. On the bottom PMT, an additional small mirror is placed to increase the sensitivity of photons with only a few scattering processes. Figure 1 shows the full logger with all components. 
Most of the development and design have been done prior to the first measurement season and can be read up in previous works [12]. Only the light source was altered between the two measurement seasons. The light source is based on flasher boards with one LED each. In the two measurement seasons four flasher boards with wavelengths of $245 \mathrm{~nm}, 278 \mathrm{~nm}, 310 \mathrm{~nm}$ and $370 \mathrm{~nm}$ were used. The nanosecond light pulses are obtained using a Kapustinsky Pulser with adjustible light intensity. In the first measurement season an integrating sphere [13] was used to create a well-defined emission profile. For the second measurement season the integrating sphere was removed to increase the number of emitted photons.

\section{Measurements}

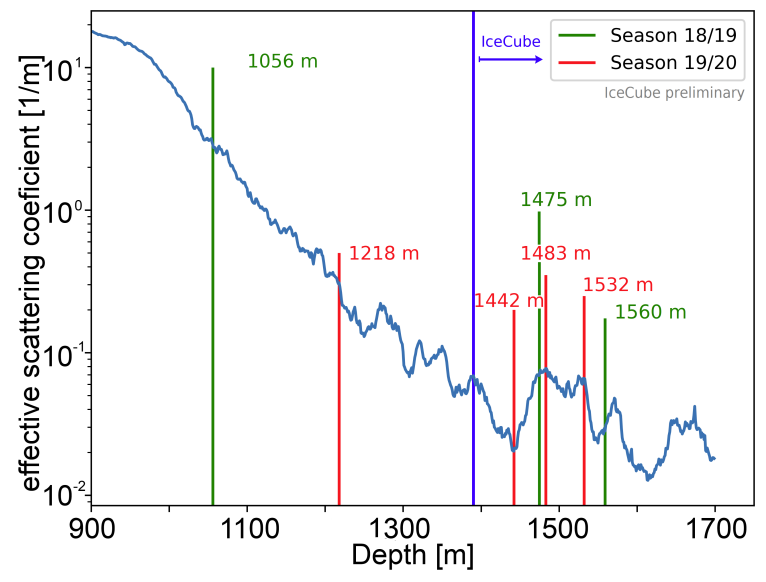

Figure 3: All measurement depths of the two seasons, together with the effective scattering coefficients[7], shifted to compensate the ice tilt between IceCube and SPICEcore. Depending on the depth the error of the ice tilt can increase up to $30 \mathrm{~m}$.

\subsection{Second measurement season}

The second measurement was performed in the austral summer 2019/2020. In total 4 measurement days were taken with three different flasher boards, where the flasher board with $250 \mathrm{~nm}$ sons with a total of 4 wavelengths at 7 depths in the ice. Figure 3 shows the measurement depths together with the expected scattering coefficients.

\subsection{First measurement season}

In the austral summer 2018/2019 the first data set was collected on two days, at depths of $1056 \mathrm{~m}, 1475 \mathrm{~m}$, and $1560 \mathrm{~m}$, using both the $278 \mathrm{~nm}$ and $400 \mathrm{~nm}$ LED at each depth. Due to light intensity problems only the $278 \mathrm{~nm}$ LED provided useful data. During the whole measurement season one of the PMT channels, connected to a wavelength shifting rod did not record data. For some measurements the open PMTs picked up electric noise from the light source, but in every measurement at least 3 Channels recorded useful data.
The measurements were done in two sea- 

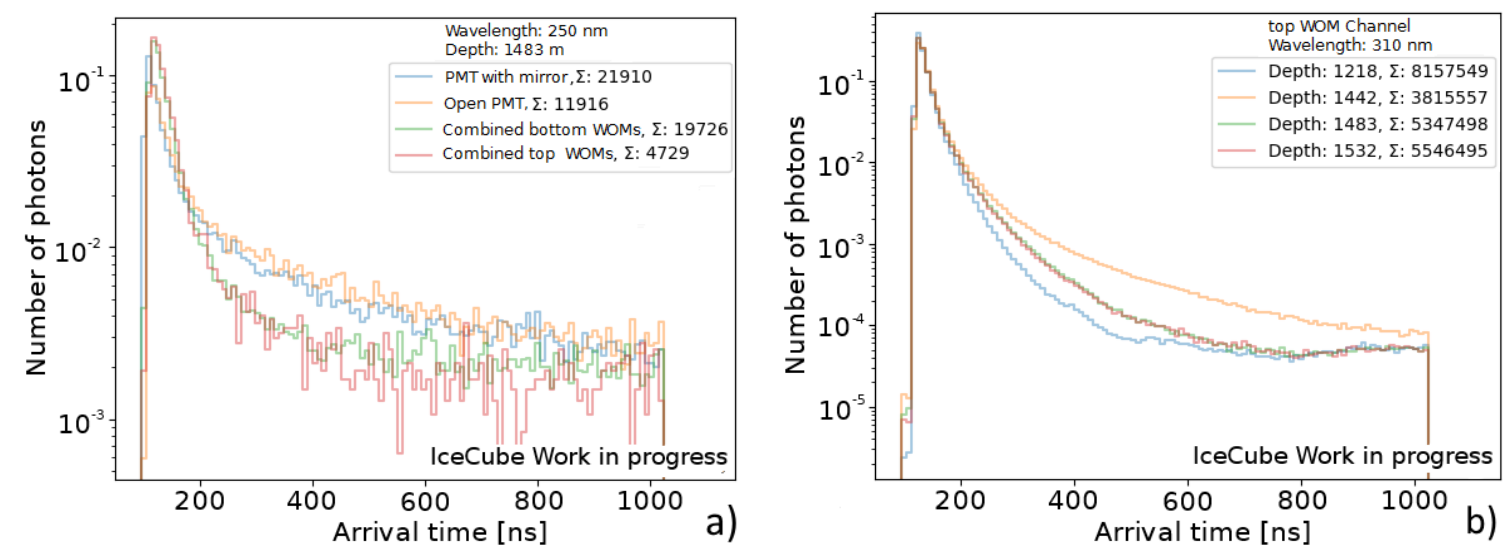

Figure 4: Prepared example data sets of the measurements a) with $250 \mathrm{~nm}$ at $1483 \mathrm{~m}$ depth for all channels and b) with $310 \mathrm{~nm}$ and the top WOM channel for all measured depths.

was used on two measurement days. The measurements were done at depths of $1218 \mathrm{~m}, 1442 \mathrm{~m}$, $1483 \mathrm{~m}$ and $1532 \mathrm{~m}$.

The measurements with $250 \mathrm{~nm}, 310 \mathrm{~nm}$ and $370 \mathrm{~nm}$ all provided useful data. For the $250 \mathrm{~nm}$ measurement one channel connected to a wavelength shifting rod was not working.

\subsection{Data preparation}

To prepare the data for analysis it is represented in the form of histograms with $8 \mathrm{~ns}$ bins (limited by a firmware bug), and cut to a time window from $80 \mathrm{~ns}$ to $1050 \mathrm{~ns}$. The PMTs connected to the wavelength shifting rods are summed for each side, to have only two WOM channels, one for the bottom PMTs (the side nearer to the light source) next to the PMT with the mirror and one for the top PMTs (further away from the light source).

Figure 4 shows two sorted and prepared example datasets. (a) displays all channels of one measurement with a wavelength of $245 \mathrm{~nm}$ at a depth of $1483 \mathrm{~m}$, (b) displays the top WOM channel for all measured depths with $310 \mathrm{~nm}$. From these examples, it is evident, that the WOM channels have a larger time spread due to the wavelength shifting. Also the different depths have visible differences in the histograms.

\section{Data Analysis}

The analysis is done by comparing the experimental data to Monte Carlo (MC) simulation with different absorption and scattering coefficients. The comparison to data is done using a binned maximum likelihood fit.

\subsection{Simulation}

The simulation models the experimental design in as much detail as possible. For the light emission, angular distribution, and wavelength spectrum of the LEDs datasheet values are interpolated.

The simulation follows the light path out of the calibration device through the quartz glass and Estisol into the ice using Fresnel equations. Every photon reaching the ice is assigned an absorption 
and scattering length sampled from random exponential distributions with the absorption and scattering coefficient as coefficients. After each scattering length, a scattering angle is sampled and the photon receives a new direction and scattering length. After every scattering process, the traveled path length is integrated and compared to the absorption length. After passing the assigned absorption length in the ice the photon is counted as absorbed in the ice. The scattering angle is highly dependent on the scattering model. For the simulation, Mie-Scattering was tested, but found to be impractical, since the experiment is mostly sensitive to backward scattering. Rayleigh scattering is used instead. The angular distribution for Rayleigh scattering follows a $(1-\cos \vartheta)^{2}$-distribution, with $\vartheta$ as the scattering angle.

Photons scattered back to the detector again pass through the Estisol and quartz glass into the detector and are counted as detected when crossing a PMT or wavelength shifting rod. The transit time spread of the different detection ways was measured in the laboratory and is dependent on the position of the photon.

\subsection{Maximum Likelihood fit}

To analyze the measurements, the distribution of binned photon arrival times is compared to the simulation. The comparison is done by calculating a test statistic $T S$ for every simulation according to the formula

$$
T S=\sum_{i=1}^{N} \frac{\left(d_{i}-a_{i} \cdot N_{d} / N_{a}\right)^{2}}{d_{i}+a_{i} \cdot N_{d}^{2} / N_{a}^{2}}
$$

where $N$ is total number of bins in the measurement, $d_{i}$ and $a_{i}$ are the number of events in the bin $i$ for the measurement $d$ and the Monte-Carlo simulation $a$ and $N_{d}$ and $N_{a}$ are the total number of events in the measurement and Monte-Carlo simulation [14].

With this test statistic, a best fitting simulation with a given set of parameters can be found. Figure 5 a) shows how the data of one depth, wavelength, and PMT-Channel connected to a WOM and five simulations are matching up. Four simulations are done with a set of high or low scattering and absorption parameters to show the boundaries of the chosen 2D scan. One simulation with a set of medium coefficients is shown in red and gives the best fit with the smallest calculated $T S$. Below the time distributions, the $T S$ per bin is plotted, so to understand the influence of each part of the distribution

To find a region of trustworthy minima the simulation with the lowest $T S$ is re-simulated and analysed 100 times to find a standard deviation $\sigma$. The true value for the parameters is supposed to lie inside an area where the difference of the $T S$ values to the minimum is smaller than $\sigma$, called the $1 \sigma$ region. This method is used to compensate for the limited simulation time. Since the number of simulated photons are smaller by a factor 10 to 100 it statistical error is mostly driven by the simulation instead of the measurement. This represents only the statistical error and not the systematic errors of the measurement.

\subsection{Open issues}

The analysis returns a well defined minimum for each channel of the measurement, but there are still unsolved inconsistencies to be explained. Figure 6 a) and b) show two simplified simulation 

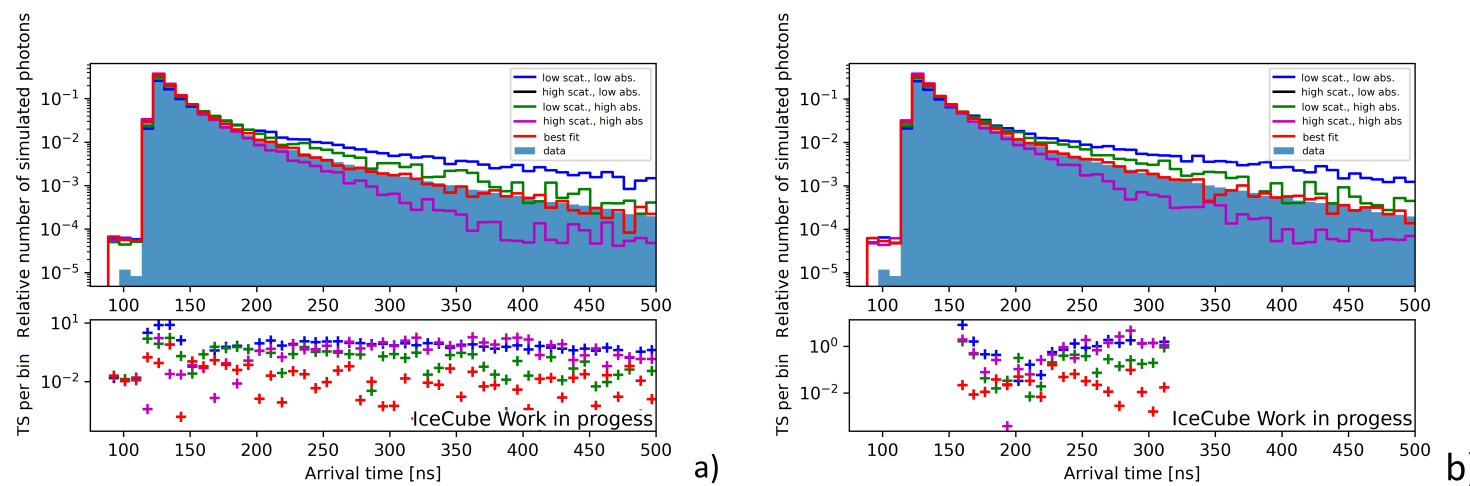

Figure 5: Dataset of a measurement with 5 simulations, 4 at the edges of the chosen parameter space and one best fit, a) for all bins with 10 or more entries, b) for a restricted time window of $150 \mathrm{~ns}-300 \mathrm{~ns}$.
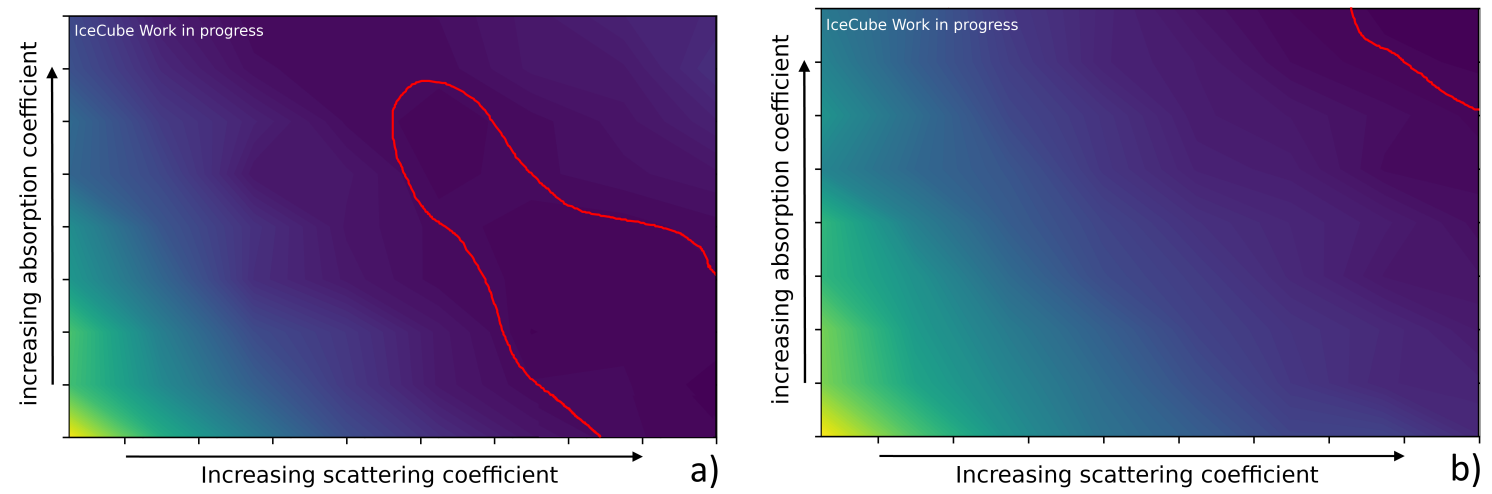

Figure 6: Simplified TS grid of several simulated sets of parameters compared to one data set for two PMT-channels of the same measurement.

grids of $T S$ calculations as a function of absorption and scattering. Both axes depict about 1 order of magnitude for each parameter. The red curve indicates the $1 \sigma$ region around the minimum.

The first unexplained observation is the differences between the PMT-channels. Comparing the minima in Figure 6 a) and b) the $\sigma$ regions are not overlapping. Therefore no definite minimum connecting all channels of one measurement has yet been found. This questions the correctness of the simulation and how well the experimental setup is understood.

Another concern is the size of the $\sigma$ region. For some measurements as 6 a) it covers almost the whole simulation grid. This and the form of the $\sigma$ region indicate a strong correlation of the two parameters. The choice of the scanned parameter space has to be therefore made very carefully to not have a minimum on the borders of the scanned area.

To decouple the two parameters the histograms are restricted to a time window of $150 \mathrm{~ns}$ $300 \mathrm{~ns}$, where the distributions are believed to be mostly absorption driven. Figure $5 \mathrm{~b}$ ) again shows the best fit and several example simulations for this restricted time window. Figure 7 gives again the simplified simulation grid with the $\sigma$ region around the minimum, showing still the same dependency of the two parameters. This leads to the conclusion that the two parameters are not easily decoupled and the final results could be a combined extinction parameter instead of independent absorption and scattering coefficients. 

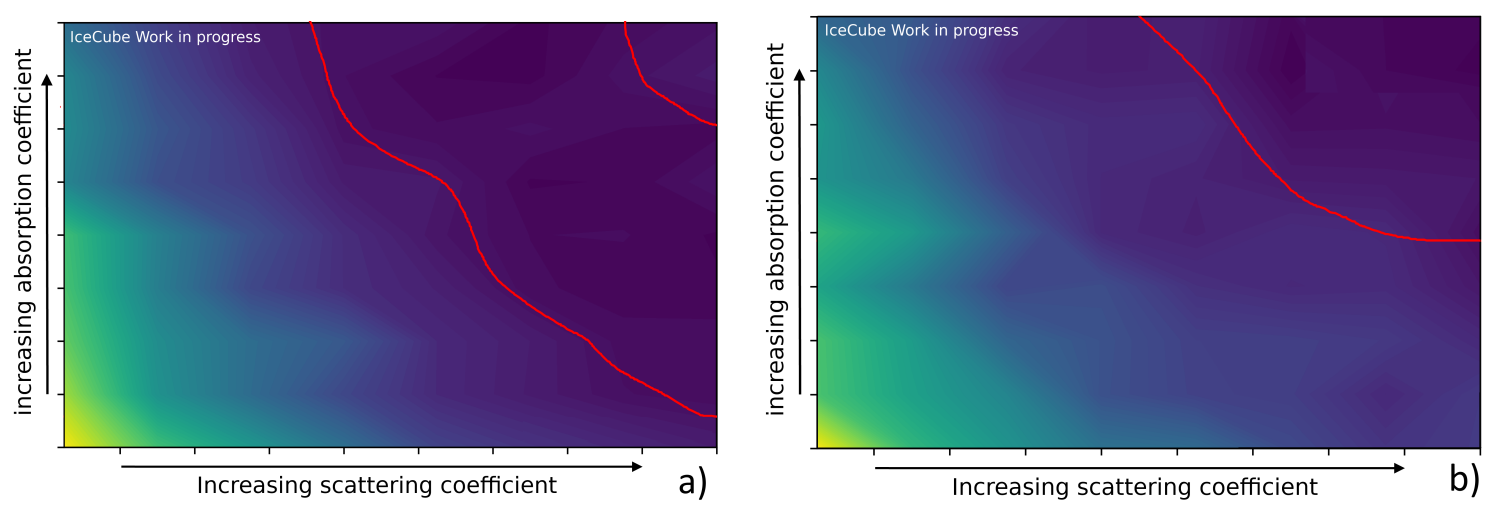

Figure 7: Simplified TS grid of several simulated sets of parameters compared to one data set for two PMT-channels of the same measurement with a restricted time window.

\section{Outlook}

In the future, the focus will be on increasing the understanding of the experimental setup to understand and compensate for the differences in the measurement channels. This should lead to a combined minimum for each data set on each measured wavelength and depth, which can be compared to previous ice calibrations.

\section{Acknowledgements}

The author would like to thank the SPICEcore collaboration for providing the borehole, the US Ice Drilling Program, the Antarctic Support Contractor and the NSF National Science Foundation for providing the equipment to perform the measurement and for their support at South Pole.

\section{References}

[1] IceCube Collaboration, M. G. Aartsen et al. JINST 12 no. 03, (2017) P03012.

[2] IceCube Collaboration PoS ICRC2017 (2017) 1052.

[3] IceCube Collaboration $P o S$ ICRC2021 (these proceedings) .

[4] Y. D. He and P. B. Price J. Geophys. Res.: Atmos 103 no. D14, (1998) 17041-17056.

[5] F. Urbach Phys. Rev. 92 no. I5, (1953) P01324.

[6] A. P. Minton JPC 75 (1971) 1162-1164.

[7] IceCube Collaboration, M. Ackermann et al. JGR 111 no. O3, (2006) .

[8] K. A. Casey et al. Annals of Glaciology 55 (1971) 137-146.

[9] IceCube Collaboration PoS ICRC2019 (July 22, 2019) 983.

[10] IceCube Collaboration $P o S$ ICRC2021 (these proceedings) .

[11] M. Rongen, R. Bay, and S. Blot The Cryosphere 14 (08, 2020) 2537-2543.

[12] IceCube Collaboration PoS ICRC2019 (2019) 847.

[13] IceCube Collaboration $P o S$ ICRC2021 (these proceedings) .

[14] B. Barlow PCP 77 no. 2, (1993) 219-228. 


\section{Full Author List: IceCube Collaboration}

R. Abbasi ${ }^{17}$, M. Ackermann ${ }^{59}$, J. Adams ${ }^{18}$, J. A. Aguilar ${ }^{12}$, M. Ahlers ${ }^{22}$, M. Ahrens ${ }^{50}$, C. Alispach ${ }^{28}$, A. A. Alves Jr. ${ }^{31}$, N. M.

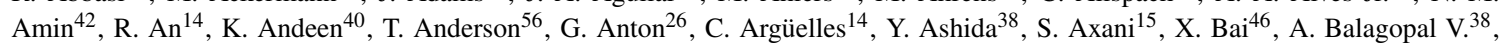
A. Barbano ${ }^{28}$, S. W. Barwick ${ }^{30}$, B. Bastian ${ }^{59}$, V. Basu ${ }^{38}$, S. Baur ${ }^{12}$, R. Bay ${ }^{8}$, J. J. Beatty ${ }^{20,21}$, K.-H. Becker ${ }^{58}$, J. Becker Tjus ${ }^{11}$, C. Bellenghi $^{27}$, S. BenZvi ${ }^{48}$, D. Berley ${ }^{19}$, E. Bernardini ${ }^{59,60}$, D. Z. Besson ${ }^{34,61}$, G. Binder ${ }^{8,9}$, D. Bindig ${ }^{58}$, E. Blaufuss ${ }^{19}$, S. Blot ${ }^{59}$, M. Boddenberg ${ }^{1}$, F. Bontempo ${ }^{31}$, J. Borowka ${ }^{1}$, S. Böser ${ }^{39}$, O. Botner ${ }^{57}$, J. Böttcher ${ }^{1}$, E. Bourbeau ${ }^{22}$, F. Bradascio ${ }^{59}$, J. Braun ${ }^{38}$, S. Bron $^{28}$, J. Brostean-Kaiser ${ }^{59}$, S. Browne ${ }^{32}$, A. Burgman ${ }^{57}$, R. T. Burley ${ }^{2}$, R. S. Busse ${ }^{41}$, M. A. Campana ${ }^{45}$, E. G. Carnie-Bronca ${ }^{2}$, C. $\mathrm{Chen}^{6}$, D. Chirkin ${ }^{38}$, K. Choi ${ }^{52}$, B. A. Clark ${ }^{24}$, K. Clark ${ }^{33}$, L. Classen ${ }^{41}$, A. Coleman ${ }^{42}$, G. H. Collin ${ }^{15}$, J. M. Conrad ${ }^{15}$, P. Coppin $^{13}$, P. Correa ${ }^{13}$, D. F. Cowen ${ }^{55}, 56$, R. Cross $^{48}$, C. Dappen ${ }^{1}$, P. Dave ${ }^{6}$, C. De Clercq ${ }^{13}$, J. J. DeLaunay ${ }^{56}$, H. Dembinski ${ }^{42}$, K. Deoskar $^{50}$, S. De Ridder ${ }^{29}$, A. Desai ${ }^{38}$, P. Desiati ${ }^{38}$, K. D. de Vries ${ }^{13}$, G. de Wasseige ${ }^{13}$, M. de With ${ }^{10}$, T. DeYoung ${ }^{24}$, S. Dharani ${ }^{1}$, A. Diaz ${ }^{15}$, J. C. Díaz-Vélez ${ }^{38}$, M. Dittmer ${ }^{41}$, H. Dujmovic ${ }^{31}$, M. Dunkman ${ }^{56}$, M. A. DuVernois ${ }^{38}$, E. Dvorak ${ }^{46}$, T. Ehrhardt ${ }^{39}$, P. Eller $^{27}$, R. Engel ${ }^{31,32}$, H. Erpenbeck ${ }^{1}$, J. Evans ${ }^{19}$, P. A. Evenson ${ }^{42}$, K. L. Fan ${ }^{19}$, A. R. Fazely ${ }^{7}$, S. Fiedlschuster ${ }^{26}$, A. T. Fienberg ${ }^{56}$, K. Filimonov ${ }^{8}$, C. Finley ${ }^{50}$, L. Fischer ${ }^{59}$, D. Fox ${ }^{55}$, A. Franckowiak ${ }^{11,59}$, E. Friedman ${ }^{19}$, A. Fritz ${ }^{39}$, P. Fürst ${ }^{1}$, T. K. Gaisser ${ }^{42}$, J. Gallagher $^{37}$, E. Ganster ${ }^{1}$, A. Garcia ${ }^{14}$, S. Garrappa ${ }^{59}$, L. Gerhardt ${ }^{9}$, A. Ghadimi ${ }^{54}$, C. Glaser ${ }^{57}$, T. Glauch ${ }^{27}$, T. Glüsenkamp ${ }^{26}$, A. Goldschmidt $^{9}$, J. G. Gonzalez ${ }^{42}$, S. Goswami ${ }^{54}$, D. Grant ${ }^{24}$, T. Grégoire ${ }^{56}$, S. Griswold ${ }^{48}$, M. Gündüz ${ }^{11}$, C. Günther ${ }^{1}$, C. Haack ${ }^{27}$,

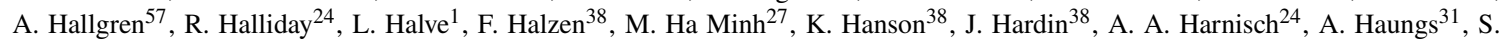

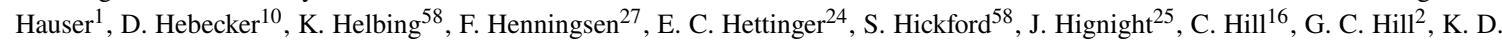

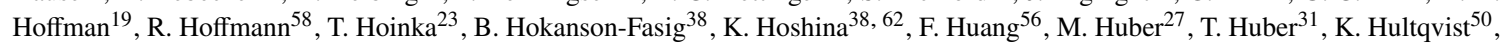
M. Hünnefeld ${ }^{23}$, R. Hussain ${ }^{38}$, S. In ${ }^{52}$, N. Iovine ${ }^{12}$, A. Ishihara ${ }^{16}$, M. Jansson ${ }^{50}$, G. S. Japaridze ${ }^{5}$, M. Jeong ${ }^{52}$, B. J. P. Jones ${ }^{4}$, D. Kang ${ }^{31}$, W. Kang ${ }^{52}$, X. Kang ${ }^{45}$, A. Kappes ${ }^{41}$, D. Kappesser ${ }^{39}$, T. Karg ${ }^{59}$, M. Karl ${ }^{27}$, A. Karle ${ }^{38}$, U. Katz ${ }^{26}$, M. Kauer ${ }^{38}$, M. Kellermann ${ }^{1}$, J. L. Kelley $^{38}$, A. Kheirandish ${ }^{56}$, K. Kin ${ }^{16}$, T. Kintscher ${ }^{59}$, J. Kiryluk ${ }^{51}$, S. R. Klein ${ }^{8,9}$, R. Koirala ${ }^{42}$, H. Kolanoski ${ }^{10}$, T. Kontrimas ${ }^{27}$, L. $\mathrm{Köpke}^{39}$, C. Kopper ${ }^{24}$, S. Kopper ${ }^{54}$, D. J. Koskinen ${ }^{22}$, P. Koundal ${ }^{31}$, M. Kovacevich ${ }^{45}$, M. Kowalski ${ }^{10,59}$, T. Kozynets ${ }^{22}$, E. Kun ${ }^{11}$, N. Kurahashi ${ }^{45}$, N. Lad ${ }^{59}$, C. Lagunas Gualda ${ }^{59}$, J. L. Lanfranchi ${ }^{56}$, M. J. Larson ${ }^{19}$, F. Lauber ${ }^{58}$, J. P. Lazar ${ }^{14,38}$, J. W. Lee ${ }^{52}$, K.

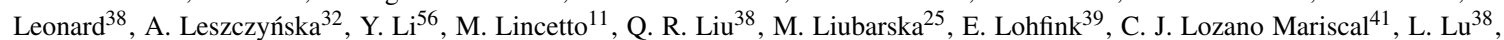

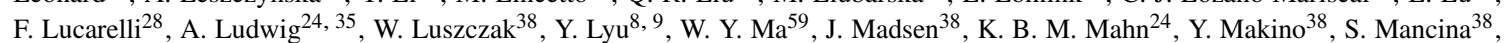
I. C. Mariş ${ }^{12}$, R. Maruyama ${ }^{43}$, K. Mase ${ }^{16}$, T. McElroy ${ }^{25}$, F. McNally ${ }^{36}$, J. V. Mead ${ }^{22}$, K. Meagher $^{38}$, A. Medina ${ }^{21}$, M. Meier ${ }^{16}$, S. Meighen-Berger $^{27}$, J. Micallef ${ }^{24}$, D. Mockler ${ }^{12}$, T. Montaruli ${ }^{28}$, R. W. Moore ${ }^{25}$, R. Morse ${ }^{38}$, M. Moulai $^{15}$, R. Naab ${ }^{59}$, R. Nagai ${ }^{16}$, U. Naumann $^{58}$, J. Necker ${ }^{59}$, L. V. Nguyễn ${ }^{24}$, H. Niederhausen ${ }^{27}$, M. U. Nisa ${ }^{24}$, S. C. Nowicki ${ }^{24}$, D. R. Nygren ${ }^{9}$, A. Obertacke Pollmann ${ }^{58}$, M. Oehler ${ }^{31}$, A. Olivas ${ }^{19}$, E. O'Sullivan ${ }^{57}$, H. Pandya ${ }^{42}$, D. V. Pankova ${ }^{56}$, N. Park ${ }^{33}$, G. K. Parker ${ }^{4}$, E. N. Paudel ${ }^{42}$, L. Paul ${ }^{40}$, C. Pérez de los $\operatorname{Heros}^{57}$, L. Peters ${ }^{1}$, J. Peterson ${ }^{38}$, S. Philippen ${ }^{1}$, D. Pieloth ${ }^{23}$, S. Pieper ${ }^{58}$, M. Pittermann ${ }^{32}$, A. Pizzuto ${ }^{38}$, M. Plum ${ }^{40}$, Y. Popovych $^{39}$, A. Porcellii ${ }^{29}$, M. Prado Rodriguez ${ }^{38}$, P. B. Price ${ }^{8}$, B. Pries ${ }^{24}$, G. T. Przybylski ${ }^{9}$, C. Raab ${ }^{12}$, A. Raissi ${ }^{18}$, M. Rameez ${ }^{22}$, K. Rawlins $^{3}$, I. C. Rea ${ }^{27}$, A. Rehman ${ }^{42}$, P. Reichherzer ${ }^{11}$, R. Reimann ${ }^{1}$, G. Renzi ${ }^{12}$, E. Resconi ${ }^{27}$, S. Reusch ${ }^{59}$, W. Rhode ${ }^{23}$, M. Richman ${ }^{45}$, B. Riedel ${ }^{38}$, E. J. Roberts ${ }^{2}$, S. Robertson ${ }^{8,9}$, G. Roellinghoff ${ }^{52}$, M. Rongen ${ }^{39}$, C. Rott ${ }^{49}, 52$, T. Ruhe ${ }^{23}$, D. Ryckbosch ${ }^{29}$, D. Rysewyk $\mathrm{Cantu}^{24}$, I. Safa ${ }^{4,}{ }^{38}$, J. Saffer ${ }^{32}$, S. E. Sanchez Herrera ${ }^{24}$, A. Sandrock ${ }^{23}$, J. Sandroos ${ }^{39}$, M. Santander ${ }^{54}$, S. Sarkar ${ }^{44}$, S. Sarkar ${ }^{25}$, K. Satalecka $^{59}$, M. Scharf ${ }^{1}$, M. Schaufel ${ }^{1}$, H. Schieler ${ }^{31}$, S. Schindler ${ }^{26}$, P. Schlunder ${ }^{23}$, T. Schmidt ${ }^{19}$, A. Schneider ${ }^{38}$, J. Schneider ${ }^{26}$, F. G. Schröder ${ }^{31,42}$, L. Schumacher ${ }^{27}$, G. Schwefer ${ }^{1}$, S. Sclafani ${ }^{45}$, D. Seckel ${ }^{42}$, S. Seunarine ${ }^{47}$, A. Sharma ${ }^{57}$, S. Shefali ${ }^{32}$, M. Silva $^{38}$, B. Skrzypek ${ }^{14}$, B. Smithers ${ }^{4}$, R. Snihur ${ }^{38}$, J. Soedingrekso ${ }^{23}$, D. Soldin ${ }^{42}$, C. Spannfellner ${ }^{27}$, G. M. Spiczak ${ }^{47}$, C. Spiering ${ }^{59,}$, 61 , J. Stachurska $^{59}$, M. Stamatikos ${ }^{21}$, T. Stanev ${ }^{42}$, R. Stein ${ }^{59}$, J. Stettner ${ }^{1}$, A. Steuer ${ }^{39}$, T. Stezelberger ${ }^{9}$, T. Stürwald ${ }^{58}$, T. Stuttard ${ }^{22}$, G. W. Sullivan $^{19}$, I. Taboada ${ }^{6}$, F. Tenholt ${ }^{11}$, S. Ter-Antonyan ${ }^{7}$, S. Tilav ${ }^{42}$, F. Tischbein ${ }^{1}$, K. Tollefson ${ }^{24}$, L. Tomankova ${ }^{11}$, C. Tönnis ${ }^{53}$, S. Toscano $^{12}$, D. Tosi ${ }^{38}$, A. Trettin ${ }^{59}$, M. Tselengidou ${ }^{26}$, C. F. Tung ${ }^{6}$, A. Turcati ${ }^{27}$, R. Turcotte ${ }^{31}$, C. F. Turley ${ }^{56}$, J. P. Twagirayezu ${ }^{24}$, B. $\mathrm{Ty}^{38}$, M. A. Unland Elorrieta ${ }^{41}$, N. Valtonen-Mattila ${ }^{57}$, J. Vandenbroucke ${ }^{38}$, N. van Eijndhoven ${ }^{13}$, D. Vannerom ${ }^{15}$, J. van Santen $^{59}, \mathrm{~S}$.

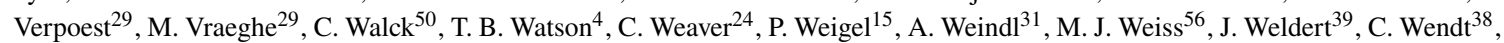
J. Werthebach ${ }^{23}$, M. Weyrauch ${ }^{32}$, N. Whitehorn ${ }^{24,35}$, C. H. Wiebusch ${ }^{1}$, D. R. Williams ${ }^{54}$, M. Wolf ${ }^{27}$, K. Woschnagg ${ }^{8}$, G. Wrede $^{26}$, J.

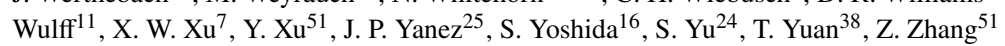

${ }^{1}$ III. Physikalisches Institut, RWTH Aachen University, D-52056 Aachen, Germany

${ }^{2}$ Department of Physics, University of Adelaide, Adelaide, 5005, Australia

${ }^{3}$ Dept. of Physics and Astronomy, University of Alaska Anchorage, 3211 Providence Dr., Anchorage, AK 99508, USA

${ }^{4}$ Dept. of Physics, University of Texas at Arlington, 502 Yates St., Science Hall Rm 108, Box 19059, Arlington, TX 76019, USA

${ }^{5}$ CTSPS, Clark-Atlanta University, Atlanta, GA 30314, USA

${ }^{6}$ School of Physics and Center for Relativistic Astrophysics, Georgia Institute of Technology, Atlanta, GA 30332, USA

${ }^{7}$ Dept. of Physics, Southern University, Baton Rouge, LA 70813, USA

${ }^{8}$ Dept. of Physics, University of California, Berkeley, CA 94720, USA

${ }^{9}$ Lawrence Berkeley National Laboratory, Berkeley, CA 94720, USA

${ }^{10}$ Institut für Physik, Humboldt-Universität zu Berlin, D-12489 Berlin, Germany

${ }^{11}$ Fakultät für Physik \& Astronomie, Ruhr-Universität Bochum, D-44780 Bochum, Germany

${ }^{12}$ Université Libre de Bruxelles, Science Faculty CP230, B-1050 Brussels, Belgium

${ }^{13}$ Vrije Universiteit Brussel (VUB), Dienst ELEM, B-1050 Brussels, Belgium

${ }^{14}$ Department of Physics and Laboratory for Particle Physics and Cosmology, Harvard University, Cambridge, MA 02138, USA

${ }^{15}$ Dept. of Physics, Massachusetts Institute of Technology, Cambridge, MA 02139, USA 
${ }^{16}$ Dept. of Physics and Institute for Global Prominent Research, Chiba University, Chiba 263-8522, Japan

${ }^{17}$ Department of Physics, Loyola University Chicago, Chicago, IL 60660, USA

${ }^{18}$ Dept. of Physics and Astronomy, University of Canterbury, Private Bag 4800, Christchurch, New Zealand

19 Dept. of Physics, University of Maryland, College Park, MD 20742, USA

${ }^{20}$ Dept. of Astronomy, Ohio State University, Columbus, OH 43210, USA

${ }^{21}$ Dept. of Physics and Center for Cosmology and Astro-Particle Physics, Ohio State University, Columbus, OH 43210, USA

${ }^{22}$ Niels Bohr Institute, University of Copenhagen, DK-2100 Copenhagen, Denmark

${ }^{23}$ Dept. of Physics, TU Dortmund University, D-44221 Dortmund, Germany

${ }^{24}$ Dept. of Physics and Astronomy, Michigan State University, East Lansing, MI 48824, USA

${ }^{25}$ Dept. of Physics, University of Alberta, Edmonton, Alberta, Canada T6G 2E1

${ }^{26}$ Erlangen Centre for Astroparticle Physics, Friedrich-Alexander-Universität Erlangen-Nürnberg, D-91058 Erlangen, Germany

${ }^{27}$ Physik-department, Technische Universität München, D-85748 Garching, Germany

${ }^{28}$ Département de physique nucléaire et corpusculaire, Université de Genève, CH-1211 Genève, Switzerland

${ }^{29}$ Dept. of Physics and Astronomy, University of Gent, B-9000 Gent, Belgium

${ }^{30}$ Dept. of Physics and Astronomy, University of California, Irvine, CA 92697, USA

${ }^{31}$ Karlsruhe Institute of Technology, Institute for Astroparticle Physics, D-76021 Karlsruhe, Germany

${ }^{32}$ Karlsruhe Institute of Technology, Institute of Experimental Particle Physics, D-76021 Karlsruhe, Germany

${ }^{33}$ Dept. of Physics, Engineering Physics, and Astronomy, Queen's University, Kingston, ON K7L 3N6, Canada

${ }^{34}$ Dept. of Physics and Astronomy, University of Kansas, Lawrence, KS 66045, USA

${ }^{35}$ Department of Physics and Astronomy, UCLA, Los Angeles, CA 90095, USA

${ }^{36}$ Department of Physics, Mercer University, Macon, GA 31207-0001, USA

${ }^{37}$ Dept. of Astronomy, University of Wisconsin-Madison, Madison, WI 53706, USA

${ }^{38}$ Dept. of Physics and Wisconsin IceCube Particle Astrophysics Center, University of Wisconsin-Madison, Madison, WI 53706, USA

${ }^{39}$ Institute of Physics, University of Mainz, Staudinger Weg 7, D-55099 Mainz, Germany

${ }^{40}$ Department of Physics, Marquette University, Milwaukee, WI, 53201, USA

${ }^{41}$ Institut für Kernphysik, Westfälische Wilhelms-Universität Münster, D-48149 Münster, Germany

42 Bartol Research Institute and Dept. of Physics and Astronomy, University of Delaware, Newark, DE 19716, USA

${ }^{43}$ Dept. of Physics, Yale University, New Haven, CT 06520, USA

${ }^{44}$ Dept. of Physics, University of Oxford, Parks Road, Oxford OX1 3PU, UK

${ }^{45}$ Dept. of Physics, Drexel University, 3141 Chestnut Street, Philadelphia, PA 19104, USA

${ }^{46}$ Physics Department, South Dakota School of Mines and Technology, Rapid City, SD 57701, USA

${ }^{47}$ Dept. of Physics, University of Wisconsin, River Falls, WI 54022, USA

${ }^{48}$ Dept. of Physics and Astronomy, University of Rochester, Rochester, NY 14627, USA

${ }^{49}$ Department of Physics and Astronomy, University of Utah, Salt Lake City, UT 84112, USA

${ }^{50}$ Oskar Klein Centre and Dept. of Physics, Stockholm University, SE-10691 Stockholm, Sweden

${ }^{51}$ Dept. of Physics and Astronomy, Stony Brook University, Stony Brook, NY 11794-3800, USA

52 Dept. of Physics, Sungkyunkwan University, Suwon 16419, Korea

${ }^{53}$ Institute of Basic Science, Sungkyunkwan University, Suwon 16419, Korea

${ }^{54}$ Dept. of Physics and Astronomy, University of Alabama, Tuscaloosa, AL 35487, USA

${ }^{55}$ Dept. of Astronomy and Astrophysics, Pennsylvania State University, University Park, PA 16802, USA

${ }^{56}$ Dept. of Physics, Pennsylvania State University, University Park, PA 16802, USA

57 Dept. of Physics and Astronomy, Uppsala University, Box 516, S-75120 Uppsala, Sweden

${ }^{58}$ Dept. of Physics, University of Wuppertal, D-42119 Wuppertal, Germany

${ }^{59}$ DESY, D-15738 Zeuthen, Germany

${ }^{60}$ Università di Padova, I-35131 Padova, Italy

${ }^{61}$ National Research Nuclear University, Moscow Engineering Physics Institute (MEPhI), Moscow 115409, Russia

${ }^{62}$ Earthquake Research Institute, University of Tokyo, Bunkyo, Tokyo 113-0032, Japan

\section{Acknowledgements}

USA - U.S. National Science Foundation-Office of Polar Programs, U.S. National Science Foundation-Physics Division, U.S. National Science Foundation-EPSCoR, Wisconsin Alumni Research Foundation, Center for High Throughput Computing (CHTC) at the University of Wisconsin-Madison, Open Science Grid (OSG), Extreme Science and Engineering Discovery Environment (XSEDE), Frontera computing project at the Texas Advanced Computing Center, U.S. Department of Energy-National Energy Research Scientific Computing Center, Particle astrophysics research computing center at the University of Maryland, Institute for Cyber-Enabled Research at Michigan State University, and Astroparticle physics computational facility at Marquette University; Belgium - Funds for Scientific Research (FRS-FNRS and FWO), FWO Odysseus and Big Science programmes, and Belgian Federal Science Policy Office (Belspo); Germany - Bundesministerium für Bildung und Forschung (BMBF), Deutsche Forschungsgemeinschaft (DFG), Helmholtz Alliance for Astroparticle Physics (HAP), Initiative and Networking Fund of the Helmholtz Association, Deutsches Elektronen Synchrotron (DESY), and High Performance Computing cluster of the RWTH Aachen; Sweden - Swedish Research Council, Swedish Polar Research Secretariat, Swedish National Infrastructure for Computing (SNIC), and Knut and Alice Wallenberg Foundation; Australia - Australian 
Research Council; Canada - Natural Sciences and Engineering Research Council of Canada, Calcul Québec, Compute Ontario, Canada Foundation for Innovation, WestGrid, and Compute Canada; Denmark - Villum Fonden and Carlsberg Foundation; New Zealand Marsden Fund; Japan - Japan Society for Promotion of Science (JSPS) and Institute for Global Prominent Research (IGPR) of Chiba University; Korea - National Research Foundation of Korea (NRF); Switzerland - Swiss National Science Foundation (SNSF); United Kingdom - Department of Physics, University of Oxford. 